\title{
Clinical Classification for Oncology
}

National Cancer Institute

\section{Source}

National Cancer Institute. Clinical Classification for Oncology. NCI Thesaurus. Code C106297.

Tumor classification based on pre-treatment information obtained from the physical examination, radiology tests, and biopsies. 